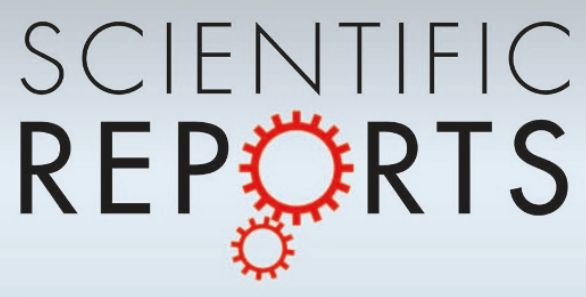

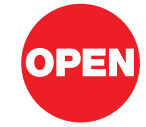

SUBJECT AREAS:

CARBON NANOTUBES

AND FULLERENES

GRAPHENE

NANOSCALE MATERIALS

CONDENSED-MATTER PHYSICS

Received

31 December 2012

Accepted

2 May 2013

Published

28 May 2013

Correspondence and requests for materials should be addressed to

A.R.H.

(aharutyunyan@ honda-ri.com)

\title{
Graphene as an atomically thin interface for growth of vertically aligned carbon
} nanotubes

\author{
Rahul Rao', Gugang Chen', Leela Mohana Reddy Arava², Kaushik Kalaga², Masahiro Ishigami', \\ Tony F. Heinz ${ }^{4}$, Pulickel M. Ajayan² \& Avetik R. Harutyunyan'
}

'Honda Research Institute USA Inc., Columbus, Ohio 43212, USA, ${ }^{2}$ Department of Mechanical Engineering and Materials Science, and Department of Chemistry, Rice University, Houston, Texas 77005, USA, ${ }^{3}$ Department of Physics and Nanoscience Technology Center, University of Central Florida, Orlando, Florida 32816, USA, ${ }^{4}$ Department of Physics and Department of Electrical

Engineering, Columbia University, New York, New York 10027, USA.

Growth of vertically aligned carbon nanotube (CNT) forests is highly sensitive to the nature of the substrate. This constraint narrows the range of available materials to just a few oxide-based dielectrics and presents a major obstacle for applications. Using a suspended monolayer, we show here that graphene is an excellent conductive substrate for CNT forest growth. Furthermore, graphene is shown to intermediate growth on key substrates, such as $\mathrm{Cu}, \mathrm{Pt}$, and diamond, which had not previously been compatible with nanotube forest growth. We find that growth depends on the degree of crystallinity of graphene and is best on mono- or few-layer graphene. The synergistic effects of graphene are revealed by its endurance after CNT growth and low contact resistances between the nanotubes and $\mathrm{Cu}$. Our results establish graphene as a unique interface that extends the class of substrate materials for CNT growth and opens up important new prospects for applications.

\footnotetext{
3 raphene and its one-dimensional counterpart, the carbon nanotube, continue to surprise, with unprecedented applications owing to their unique properties ${ }^{1-5}$. Both allotropes are also being increasingly integrated into synergistic multifunctional devices ${ }^{6-8}$. From this perspective, the recent discovery of enhanced catalytic efficiency of graphene-supported metal particles ${ }^{9,10}$ suggests a valuable approach for using graphene as a substrate for the catalytic growth of CNTs. This approach becomes even more appealing in light of the transferability of graphene and the associated possibility of graphene-mediated CNT growth on a wide variety of substrates. At present, the growth of the most popular form of CNTs, vertically aligned CNTs or forests, is restricted to a few oxide-based dielectric substrates ${ }^{11}$. A diversification of growth substrates will open new prospects for applications such as interconnects in electronics ${ }^{12}$ and next generation electrochemical devices ${ }^{13}$. For the successful application of CNTs in these vital fields, a low contact resistance between the CNTs and metal electrodes must be maintained, which can naturally be realized through direct growth of CNT forests on conductive surfaces. In this work, we establish graphene as the thinnest conductive substrate for growth of CNT forests. Furthermore, due to its transferability, we show that graphene acts as a unique interface that enables $\mathrm{CNT}$ forest growth on important surfaces that have not been possible, such as $\mathrm{Cu}, \mathrm{Pt}$ and diamond. We find that the growth of nanotubes does not destroy the graphene layer and that it exhibits a strong dependence on degree of crystallinity of graphene.
}

\section{Results}

In order to verify whether graphene can itself support vertically aligned CNT growth, we first investigated CNT growth on suspended graphene. Graphene was grown on $\mathrm{Cu}$ foil by low-pressure ${ }^{14,15}$ and atmospheric pressure ${ }^{16}$ chemical vapor deposition (CVD) using methane as the carbon source (see Methods for experimental details). The graphene layers were then transferred to a quartz substrate in which trenches had been etched. Graphene can be seen suspended over one end of an open trench in the scanning electron microscope (SEM) image in Fig. 1a. Its presence was confirmed by the redshifted Raman peaks (Fig. 1b) in the suspended regions compared to the supported regions ${ }^{17}$. Graphene exhibits two major peaks in its Raman spectrum at $\sim 1585 \mathrm{~cm}^{-1}$ ( $G$ band) and $~$ $2650 \mathrm{~cm}^{-1}\left(G^{\prime} \text { band) }\right)^{18,19}$. A third peak ( $D$ band) appears at $\sim 1330 \mathrm{~cm}^{-1}$ (with $633 \mathrm{~nm}$ excitation) due to 

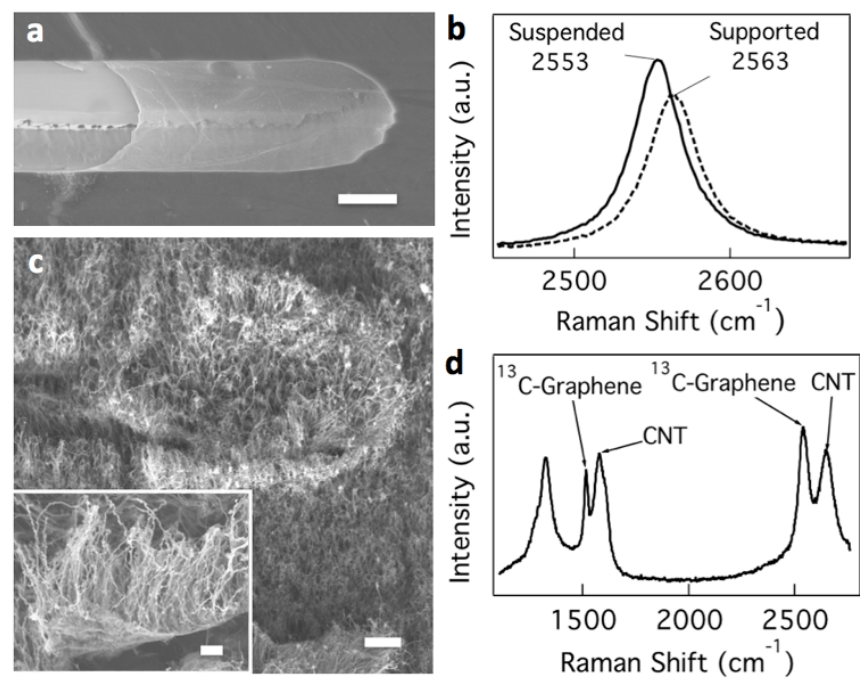

Figure $1 \mid$ CNT growth on suspended graphene. (a) SEM image showing graphene suspended across an open trench in a quartz substrate.

(b) Raman spectra in the $G^{\prime}$ band region (633 nm excitation) collected from suspended (solid trace) and supported (dashed trace) graphene. The frequencies of the peaks are listed. (c) SEM image collected from the trenched quartz substrate after CNT growth. The inset shows vertically aligned CNTs on graphene that lifted off the quartz substrate. (d) Raman spectrum collected from the trenched quartz substrate after CNT growth. The peaks of the ${ }^{13} \mathrm{C}$-graphene and CNTs can be clearly distinguished. The scale bars in (a) and (c) are $2 \mu \mathrm{m}$, and the scale bar in the inset of (c) is $200 \mathrm{~nm}$.

disorder. CNT growth on the suspended graphene was performed at $750^{\circ} \mathrm{C}$ using a floating catalyst CVD method with ferrocene and xylene as the catalyst and carbon source, respectively (Methods). Since CNTs also exhibit Raman peaks with frequencies similar to those in graphene, it is difficult to distinguish between the Raman peaks of graphene and CNTs. To circumvent this issue, ${ }^{13} \mathrm{C}$ isotope labeled methane (100\% concentration, hereafter called ${ }^{13} \mathrm{C}$-graphene) was used to synthesize the graphene on $\mathrm{Cu}$ (Graphene Laboratories Inc.) and to prepare the suspended graphene. The Raman peaks of ${ }^{13} \mathrm{C}$-graphene are redshifted compared to the usual ${ }^{12} \mathrm{C}$ Raman peaks (ref. 20 and Fig. S1) according to the following formula

$$
\omega=\omega_{12} \sqrt{\frac{m_{12}}{n_{12} m_{12}+n_{13} m_{13}}}
$$

where $\omega_{12}$ is the Raman peak frequency of ${ }^{12} \mathrm{C}$-graphene, $n_{12}$ and $n_{13}$ are the atomic fractions, and $m_{12}$ and $m_{13}$ are the atomic masses of ${ }^{12} \mathrm{C}$ and ${ }^{13} \mathrm{C}$, respectively. Fig. 1c shows an SEM image collected from the trenched quartz substrate after 2 minutes of growth. While it is not easy to visualize the graphene, the increased density of CNTs highlighting the perimeter of the trench (Fig. 1c) strongly suggests that CNTs grew on the suspended graphene. Aligned CNTs were also observed on other regions of the quartz substrates where the graphene had pulled off with the CNTs attached (Inset in Fig. 1c and Figs. S2a and S2b). The presence of graphene was verified directly by Raman spectroscopy using ${ }^{13} \mathrm{C}$-graphene with its shifted $G$ and $G^{\prime}$ bands (Fig. 1d).

We have also explored the possibility of using graphene as an intermediate to promote forest growth on other substrates by initially covering them with graphene. A natural choice to test this approach is $\mathrm{Cu}$, since the production of large-area graphene on $\mathrm{Cu}$ foil by CVD is well established ${ }^{14}$. For this purpose, we first performed graphene growth on $\mathrm{Cu}$ foil followed by CNT growth via floating catalyst CVD (Methods). As can be seen in the optical microscope image in Fig. 2a, the graphene-covered $\mathrm{Cu}$ foil exhibits dense CNT growth in sharp contrast to negligible growth on plain $\mathrm{Cu}$. The corresponding SEM image of the Cu foil after CNT growth (Fig. 2b) reveals an $80 \mu \mathrm{m}$ tall CNT forest, with vertical alignment (inset). The nanotubes produced were multi-walled (MWNTs) with an average diameter of $25 \mathrm{~nm}$ (Fig. S4).

To test whether graphene can intermediate growth on surfaces other than $\mathrm{Cu}$, we transferred CVD graphene from $\mathrm{Cu}$ to polycrystalline platinum foil using the standard graphene transfer techniques (see ref. 21 and Methods for details). Pt substrates are typically not amenable to CNT forest growth, as evidenced by the sparse CNT growth on unmodified Pt foils (Fig. 2c). However, we grew vertically aligned CNTs on graphene-covered Pt (Fig. 2d) under growth conditions analogous to those for $\mathrm{Cu}$. Furthermore, by transferring graphene to CVD diamond films and growing vertically aligned CNTs (Figs. 2e and 2f), we were able to synthesize a unique hybrid of three carbon allotropes, an advance that holds great potential for thermal management ${ }^{3}$. We note that our growth process was optimized for $\mathrm{Cu}$ substrates and that CNT forests with uniform densities could be grown on $\mathrm{Pt}$ and diamond with further optimization. Nevertheless, the successful application of graphene as a growth intermediate on both Pt (a metal) and diamond (an insulator) implies that it should be possible to grow vertically aligned CNTs on an almost unlimited range of substrates, constrained only by the CNT growth conditions. Our results also hint at the possibility that the previously reported growth of nanotube forests on inconel alloys ${ }^{22,23}$ could actually have been enabled by graphene layers formed in situ during CNT growth.

To investigate the evolution of the state of the graphene during CNT forest growth we again used ${ }^{13} \mathrm{C}$-graphene (containing $80 \%{ }^{13} \mathrm{C}$ isotope) on $\mathrm{Cu}$ foil. CNT growths were conducted for 5 and 10 minutes, followed by Raman spectroscopy analysis. Even after 10 minutes of CNT growth, the presence of the ${ }^{13} \mathrm{C}$-graphene peaks at $1535 \mathrm{~cm}^{-1}$ and $2575 \mathrm{~cm}^{-1}$ (Fig. $2 \mathrm{~g}$ ) clearly confirm that the graphene layer remains intact. Still longer synthesis times resulted in longer and denser tubes, rendering it difficult to identify Raman signals from graphene above the CNT Raman response. As separate confirmation, we performed experiments in which the CNTs were removed from the graphene-Cu substrate and the substrate was reused for CNT growth. Fig. 2h shows an SEM image of a substrate where the CNTs grown for $30 \mathrm{~min}$. have been partially removed by gently scraping. The Raman spectrum from the graphene in the cleaned region (Figs. S5a and S5b) exhibited broader peaks with a higher $D$ band intensity, indicative of higher defect density compared to its original state. We attribute these changes to the high temperature annealing process and deposition of some disordered carbon during CNT growth. In spite of this increased disorder, we were able to re-grow vertically aligned MWNTs after cleaning the substrate (Fig. $2 \mathrm{~h}$ inset and Fig. S5c), proving that the graphene retains its ability to support growth of CNT forests.

The results presented above were all relied on the use of monolayer graphene to mediate CNT growth. We now discuss the impact of the number of graphene layers on the growth process. We first decided to test this by performing CNT growth on few-layer graphene (FLG). For this purpose, we chose nickel substrates, which are known to produce high-quality $\mathrm{FLG}^{24}$. Graphene growth was performed on $\mathrm{Ni}$ foils at low-pressure using conditions similar to $\mathrm{Cu}$ (Methods). Analysis of graphene on Ni by Raman spectroscopy confirmed the presence of FLG (typically 2 - 5 layers) over most of the Ni surface (Fig. S6). While it was not easy to reliably produce known number of graphene layers on $\mathrm{Ni}$ with reasonably large homogeneous area, it is still instructive to compare CNT growth results on monolayer graphene (on $\mathrm{Cu}$ ) with FLG (on Ni). As can be seen in Figs. 3a and S7, vertically aligned CNT growth was not observed anywhere on the graphene-covered $\mathrm{Ni}$ foil. A few islands exhibiting low-density spaghetti-like tubes were observed, with a higher tube density present in areas with fewer graphene layers. Fig. 3a shows a region of the $\mathrm{Ni}$ foil where multiple graphene layers have spontaneously lifted off the foil. We explain this result as reflecting the growth of dense CNTs 

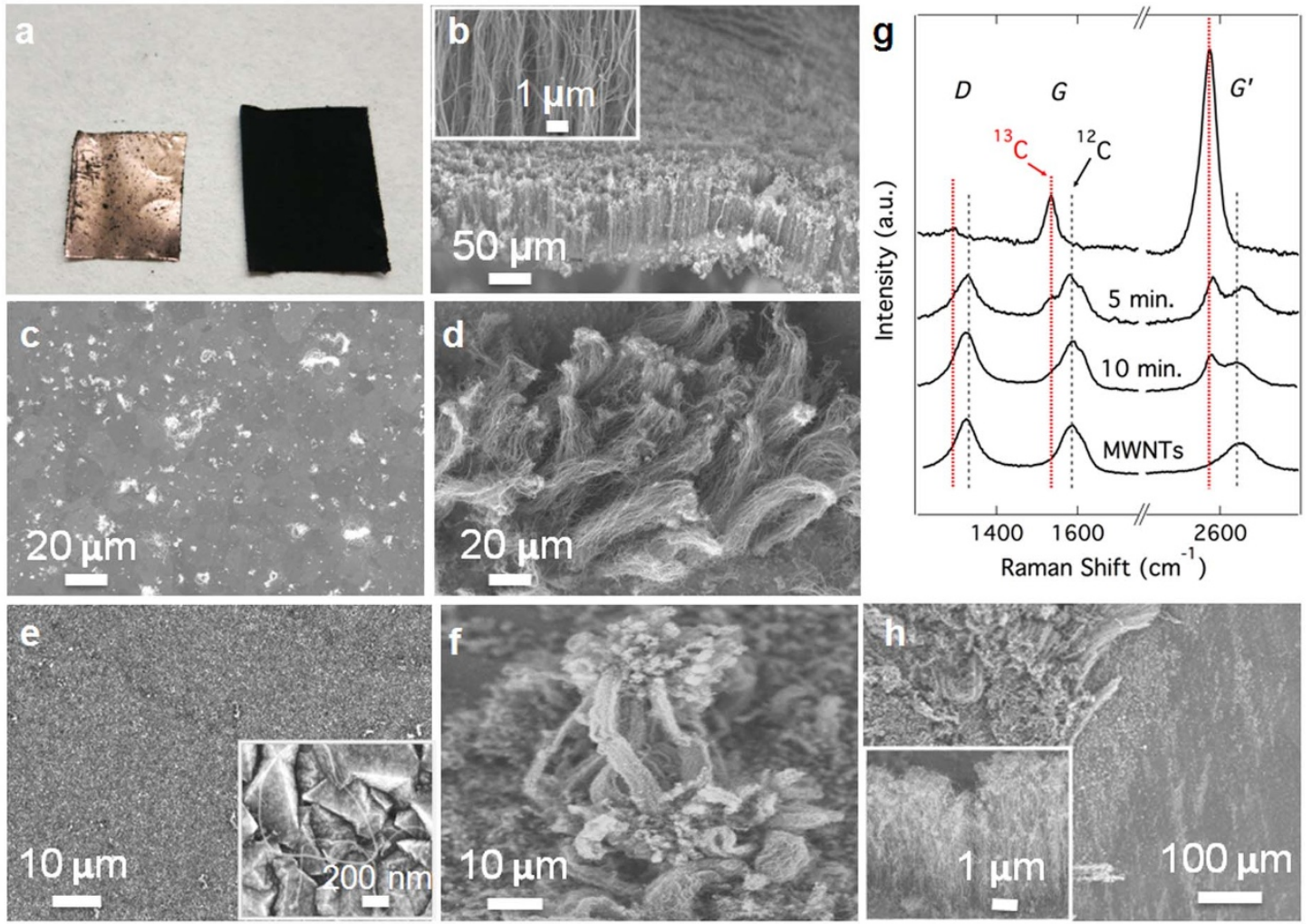

Figure $2 \mid$ CNT growth on graphene-covered surfaces. (a) Optical image of plain Cu (left) and graphene-covered Cu (right) after CNT growth. (b) SEM image of a CNT forest grown on graphene-covered Cu foil. The inset shows the vertical alignment of the CNTs. (c) SEM image collected after CNT growth on a bare Pt foil. (d), SEM image showing vertically aligned CNTs on graphene-covered Pt. (e) SEM image collected from bare diamond film after CNT growth. The inset shows a high magnification view of the diamond film with very sparse CNT coverage. (f) SEM image of vertically aligned CNTs on graphene-covered diamond. (g) Raman spectra collected from the CNTs grown on ${ }^{13} \mathrm{C}$-graphene for various growth times. The Raman peaks corresponding to both ${ }^{13} \mathrm{C}$-graphene and CNTs can be seen even after 10 minutes of growth, indicating that graphene survives the CNT growth process. (h) SEM image collected from a Cu sample from which MWNTs have been partially removed. The inset shows an SEM image of a CNT forest on Cu after re-growth.
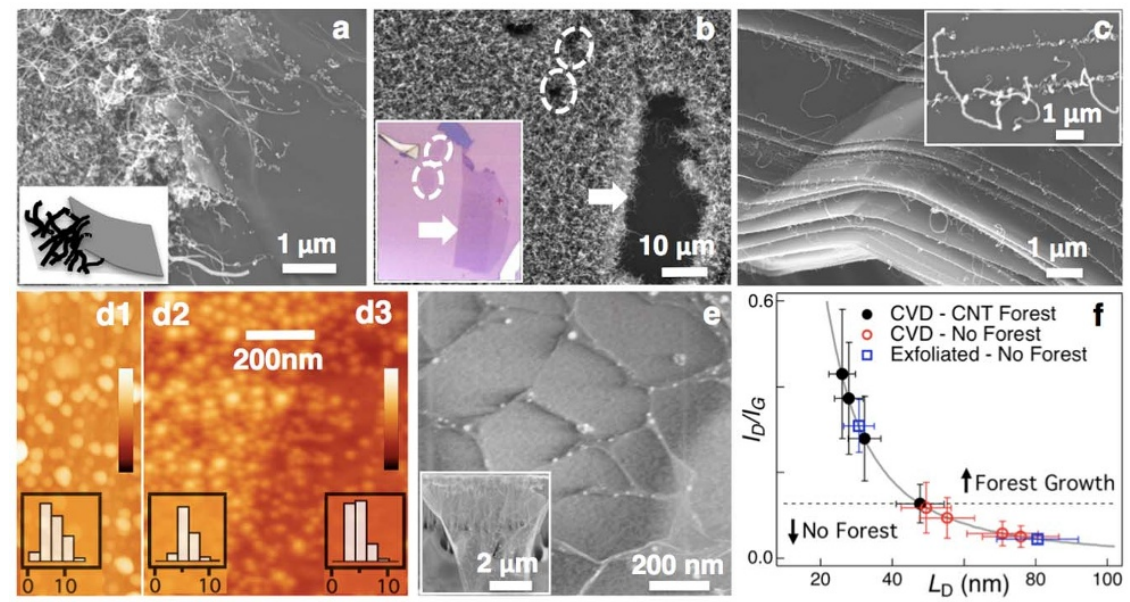

Figure 3 Dependence of number of layers and degree of crystallinity of graphene on CNT growth. (a) SEM image showing graphene layers that pulled off a Ni substrate and allowed dense CNT growth underneath (schematic drawn in inset). (b) SEM image after CNT growth and corresponding optical image before growth (inset) from exfoliated monolayer (indicated by the dashed circles) and bilayer graphene (indicated by the arrow) on $\mathrm{SiO}_{2}$ substrate. (c) SEM image collected after CNT growth on HOPG showing relatively denser growth along edges and steps (inset). AFM images of Fe nanoparticles on (d1) CVD graphene-covered $\mathrm{SiO}_{2}$, (d2) bare $\mathrm{SiO}_{2}$, and (d3) exfoliated graphene-covered $\mathrm{SiO}_{2}$. The vertical scale bars in (d1) and (d3) correspond to a height ranges from 0-50 nm and 0-20 nm, respectively. The insets in (d1), (d2) and (d3) show the particle height histograms (counts vs. height in $\mathrm{nm}$ ) obtained from AFM analysis. (e) SEM image of Fe nanoparticles on suspended CVD graphene. The inset shows a low magnification view. (f) $I_{D} / I_{G}$ values from several CVD and exfoliated graphene samples as a function of the average distance $L_{\mathrm{D}}$ between defects. 
from the remaining thinner layer of graphene on the Ni surface (See Fig. S7 for another example).

In addition to CVD graphene on $\mathrm{Cu}$ and $\mathrm{Ni}$, we performed CNT growth on mono-, bi-, and few-layer graphene on $\mathrm{Si} / \mathrm{SiO}_{2}$ substrates prepared by mechanical exfoliation from kish graphite, as well as on highly oriented pyrolytic graphite (HOPG). Remarkably, there was no CNT growth observed on either mono- or bi-layer graphene, as can be clearly seen in the optical (inset) and SEM images in Fig. 3b. The growth of CNTs on HOPG was very limited, even after long growth times (60 minutes). The CNTs were observed primarily at steps, edges, and defects (Fig. 3c), presumably because of migration of the Fe particles to these sites (inset in Fig. 3c).

\section{Discussion}

One of the distinguishing features between CVD and exfoliated graphene samples is the higher degree of crystallinity in the exfoliated material (see Fig. S8 for Raman spectra from the mono- and bi-layer graphene areas in Fig. $3 \mathrm{~b}$ ). This difference suggests that the interaction energy between the catalyst particles and the graphene is influenced by its structure. We analyzed the size and density distribution of Fe nanoparticles deposited on graphene monolayers prepared by both mechanical exfoliation and CVD (and supported on $\mathrm{SiO}_{2}$ substrates). The particles were produced by reducing the CNT growth times to a few seconds. This resulted in the formation of small Fe nanoparticles, but little growth of nanotubes. Atomic force microscopy (AFM) analysis revealed higher densities on CVD graphenecovered $\mathrm{SiO}_{2}$ (Fig. 3d1) and bare $\mathrm{SiO}_{2}$ (Fig. 3d2), with a slightly higher mean particle height in the CVD graphene (insets in Fig. $3 \mathrm{~d} 1$ and $3 \mathrm{~d} 2)^{25}$. However, the density of Fe nanoparticles (and CNTs) on exfoliated graphene (Fig. 3d3) was significantly less compared to the other two (Figs. $3 \mathrm{~d} 1$ and $3 \mathrm{~d} 2$ ). While further detailed mechanistic studies are surely warranted, the evidence suggests the differences in particle distributions between exfoliated and CVD graphene can be attributed to the greater stabilization of these particles on CVD graphene by defects or additional stresses that arise during sample transfer. Indeed, we also observed Fe nanoparticles forming a pattern on suspended graphene, possibly following the graphene grain boundaries (Fig. 3e). Deliberate control of such defects and distortions provides a promising avenue for sensitive tuning of the catalyst particle distribution and properties ${ }^{26}$. This approach could not only enhance the adsorption and reactivity of the catalyst ${ }^{27}$, but could also provide a means of influencing the size of the catalyst ${ }^{28}$.

To further investigate the impact of graphene crystallinity on the formation of CNTs, we performed CNT growth on CVD graphene samples with varying degrees of disorder based on their Raman spectra. The ratio of intensities of the $D$ band to the $G$ band $\left(I_{D} / I_{G}\right)$ is commonly used as a measure of the degree of disorder in the graphene ${ }^{18}$, with the $I_{D} / I_{G}$ related inversely to the average distance between defects $\left(L_{\mathrm{D}}\right)^{29}$. We investigated several (CVD-grown) graphene samples with $I_{D} / I_{G}$ ratios ranging from 0.045 to 0.45 , which were prepared under different growth conditions (both atmospheric and low-pressure). Surprisingly, we found that CNT forest growth is not guaranteed on all CVD-grown graphene samples. The success of CNT growth is related to the quality of the sample, implying a window for the degree of crystallinity within which graphene induces vertically aligned CNT growth. A plot of the $I_{D} / I_{G}$ ratios from several CVD and exfoliated graphene samples as a function of $L_{\mathrm{D}}$ is shown in Fig. 3f. Interestingly, the best samples for CNT forest growth exhibited higher degrees of disorder $\left(I_{D} / I_{G}>0.12\right)$, while graphene samples with less disorder $\left(I_{D} / I_{G}<0.12\right)$ did not lead to CNT forest growth. Note, however, that the data presented in Fig. 3f only considers Raman-active defects; other kinds of defects and disorder, such as zigzag edges, charged impurities and strain, do not contribute to the $D$ band intensity. In addition, exfoliated graphene with an $I_{D} / I_{G}$ ratio within the preferred window still did not induce growth of a
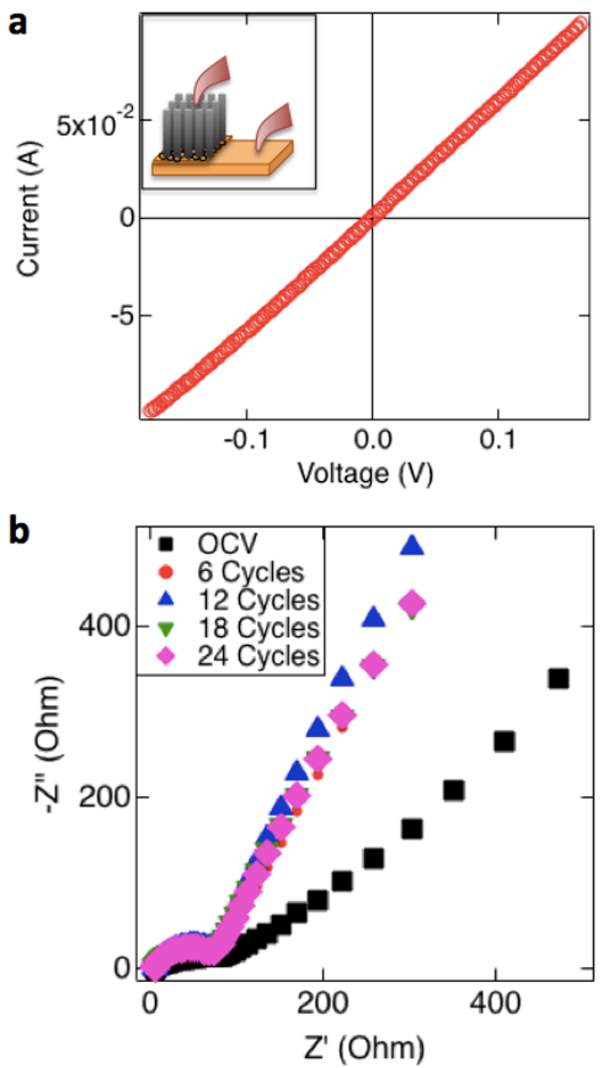

Figure 4 | Electrical characterization of CNTs on graphene-covered Cu. (a) Two-probe I-V characteristic measured between the CNT forest and $\mathrm{Cu}$ foil (schematic in inset) showing linear (Ohmic) behavior. (b) Nyquist plot of EIS spectra collected from an electrochemical cell after 6, 12, 18 and 24 cycles. The spectra retain their shape after cycling, confirming the good electrical contact between the CNTs and $\mathrm{Cu}$ electrode.

CNT forest. This indicates that there are factors other than Ramanactive defects that could influence the distribution of catalyst nanoparticles and their capacity to nucleate nanotubes. Nonetheless, the data in Fig. 3f suggests a direct relationship between the success of CNT growth and the degree of crystallinity of the graphene. We note that CNT growth was not observed on $\mathrm{Cu}$ substrates covered preliminarily by amorphous carbon; we found graphene to be necessary for enabling CNT forest growth.

Finally we examined the practical benefits of growing CNT forests on graphene-covered $\mathrm{Cu}$ foil by measuring the contact resistance between the CNTs forests and $\mathrm{Cu}$. The current-voltage (I-V) characteristic obtained from a two-probe resistivity measurement between the CNTs and $\mathrm{Cu}$ (Fig. 4a) displays Ohmic contact, with a low contact resistance $\left(\sim 50 \Omega / \mu \mathrm{m}^{2}\right)$ that is lower than previously reported values for CNT forests with similar densities $\left(\sim 10^{10} \mathrm{CNTs} /\right.$ $\left.\mathrm{cm}^{2}\right)^{22,30}$. Further evidence for the good electrical contact was obtained by electrochemical impedance spectroscopy (EIS) measurements on an electrochemical cell assembled with the CNT forests on $\mathrm{Cu}$ electrodes. The resistance of an electrochemical cell includes resistances of the electrolyte, electrodes, current collector and other internal resistances, which increase upon extended charge/discharge cycles and affect the overall performance of the cell. This in turn is reflected in the EIS measurements by an increase in the size of the semicircles upon cycling ${ }^{31,32}$. The EIS curves from our cells exhibited highly reproducible semicircular features even after 24 cycles of galvanostatic charge/discharge measurements (Fig. 4b). Similarity in shape and size of semicircles are clear indications of minimal change in resistance between the current collector $(\mathrm{Cu})$ and active electrode 
material (nanotubes) due to seamless integration through the graphene interface.

Through this work we have shown a way to transform a surface that is inactive for CNT growth to an active one by the introduction of an atomically thin intermediary, thereby dramatically extending the class of substrates for CNT growth. By doing so we have overcome a major obstacle hindering large-scale applications of CNTs and opened new prospects for the exploitation of their unique properties.

\section{Methods}

Graphene growth on $\mathrm{Cu}$ and $\mathrm{Ni}$ by low-pressure CVD. $\mathrm{Cu}$ and $\mathrm{Ni}$ foils $(25 \mu \mathrm{m}$ thick, 99.8\%, Alfa Aesar) were used as substrates for monolayer and multi-layer graphene growth, respectively. The foils were loaded into a tubular quartz furnace and purged with $\mathrm{Ar} / \mathrm{H}_{2}$ gas mixture $(4: 1)$ at a flow rate of $50 \mathrm{sccm}$ under $90 \mathrm{mTorr}$ pressure for $20 \mathrm{~min}$, followed by ramping up the furnace temperature to $1000^{\circ} \mathrm{C}$. Once the temperature was reached, it was held for $30 \mathrm{~min}$ to anneal the foils, followed by the introduction of $\mathrm{CH}_{4}(8 \mathrm{sccm}$ for $\mathrm{Cu}$ and $4 \mathrm{sccm}$ for Ni substrates) for $10 \mathrm{~min}$ along with the $\mathrm{Ar} / \mathrm{H}_{2}$ gases. Following growth, the samples were cooled down to room temperature at a rate of $30^{\circ} \mathrm{C} / \mathrm{min}$ rate under the $\mathrm{Ar} / \mathrm{H}_{2}$ mixture.

Graphene growth on $\mathrm{Cu}$ by atmospheric pressure CVD. Cu foil $(25 \mu \mathrm{m}$ thick, 99.8\% purity, Alfa Aesar) was loaded into the center of a tubular quartz furnace and heated to $1000^{\circ} \mathrm{C}$ under a constant flow of argon $(300 \mathrm{sccm})$ and hydrogen (30$100 \mathrm{sccm}$ ). Once the temperature was reached, it was held for 15 minutes to anneal the $\mathrm{Cu}$ foils, followed by the introduction of 1-2 sccm of $\mathrm{CH}_{4}$ for 30 minutes along with the $\mathrm{Ar} / \mathrm{H}_{2}$ gases. Following growth the samples were allowed to cool down to room temperature naturally.

Carbon nanotube growth by floating catalyst CVD. CNTs were grown at ambient pressure via a floating catalyst CVD method using ferrocene and xylene as the catalyst and carbon source, respectively ${ }^{33}$. Ferrocene (10 wt.\%) was dissolved in xylene through mild sonication. The mixture was then loaded into a syringe and delivered into a quartz tube furnace through a capillary connected to a syringe pump. The capillary was placed such that its exit point was just outside the hot zone of the tube furnace. The substrates (graphene-covered $\mathrm{Cu}, \mathrm{Pt}, \mathrm{SiO}_{2}$ and diamond) were loaded into the center of the quartz tube furnace, which was heated to the growth temperature of $\left(700-800^{\circ} \mathrm{C}\right)$ under a constant flow of argon $(500 \mathrm{sccm})$ and hydrogen $(60-120 \mathrm{sccm})$. After the furnace reached the growth temperature, the ferrocene/xylene mixture was injected continuously into the tube furnace at a rate of $1.2 \mathrm{ml} / \mathrm{hr}$ for the duration of the CNT growth (few seconds to 6 hours). At the end of the growth period the furnace was turned off and allowed to cool down to room temperature under the argon/hydrogen flow. The growth process produced vertically aligned multi-walled carbon nanotubes that grow via root growth on the graphenecovered substrates. The heights of the CNT forests could be controlled by the precursor injection time, with typical growth rates at $\sim 1 \mu \mathrm{m} / \mathrm{min}$. Post-growth characterization of the samples was performed with scanning electron microscopy (Zeiss Ultra 55 Plus) and micro-Raman spectroscopy (Renishaw Raman microscope, $633 \mathrm{~nm}$ excitation). To measure the $I_{D} / I_{G}$ ratios of the samples, Raman spectra were collected at 50 random spots across the samples, followed by peak fitting to calculate the average $I_{D} / I_{G}$ ratio.

Graphene transfer to arbitrary substrates. The graphene was transferred from $\mathrm{Cu}$ foils to arbitrary substrates including $\mathrm{SiO}_{2}$, Pt foils (ESPI Metals) and CVD diamond films (Delaware Diamond Knives) using a wet-chemistry polymer-based process ${ }^{21}$. PMMA (495, Micro Chem) was first spin-coated (4000 RPM for $60 \mathrm{~s}$ ) on to the graphene-covered $\mathrm{Cu}$ foils. Iron chloride (2M solution, Sigma Aldrich) was used to etch the $\mathrm{Cu}$ such that the PMMA/graphene film was floated to the surface of the solution in a petri dish. The PMMA/graphene film was then scooped up on to a silicon chip and floated into a second petri dish containing deionized water. After rinsing in the DI water for several minutes to remove the excess iron chloride, the PMMA/ graphene film was placed on to the substrate of interest and left to dry for a few hours. After drying, the substrates were heated in air to $\sim 100^{\circ} \mathrm{C}$ to enable flattening of the graphene films. Finally, the PMMA was stripped off in an acetone bath, and the samples were dried in air.

In addition to flat substrates, graphene was also transferred to patterned quartz substrates. Rectangular patterns (4-6 $\mu \mathrm{m}$ wide, $20-30 \mu \mathrm{m}$ long and $4 \mu \mathrm{m}$ deep) were fabricated on quartz substrate via e-beam lithography and graphene was subsequently transferred to make suspended graphene.

Electrical Characterization. Two-probe current-voltage (I-V) measurements. Tungsten needle probes were used for the two-probe electrical measurements, where one probe contacted the top of the CNT forest while the other probe was connected with the $\mathrm{Cu}$ foil (see inset in Fig. 4a for a schematic of the setup). The I-V measurement was performed with a Keithley 4200-SCS instrument at room temperature. spectroscopy (EIS) measurements on $\mathrm{Cu}$-graphene-CNT electrodes were conducted in a Swagelok-type cell using an AUTO LAB PGSTAT 302 potentiostat/galvanostat (Eco Chemie Utrecht, Netherlands). The test cells were assembled in an argon-filled glove box using the $\mathrm{Cu}$-graphene-CNT as working electrode, lithium metal foil as the counter/reference electrode, $1 \mathrm{M}$ solution of $\mathrm{LiPF}_{6}$ in $1: 1(\mathrm{v} / \mathrm{v})$ mixture of ethylene carbonate (EC) and dimethyl carbonate (DMC) as an electrolyte and a glass microfiber filter was used as separator. The cell was subjected to Galvanostatic charge/ discharge cycles and EIS measurements were conducted at OCV and after 6, 12, 18 and 20th cycle by applying a constant dc bias with sinusoidal signal of $10 \mathrm{mV}$ over the frequency range from $70 \mathrm{kHz}$ and $10 \mathrm{mHz}$.

1. Novoselov, K. et al. A roadmap for graphene. Nature 490, 192-200 (2012).

2. Bunch, J. S. et al. Electromechanical resonators from graphene sheets. Science 315, 490-493 (2007)

3. Balandin, A. A. Thermal properties of graphene and nanostructured carbon materials. Nat Mater 10, 569-581 (2011).

4. Avouris, P., Chen, Z. \& Perebeinos, V. Carbon-based electronics. Nature Nanotech. 2, 605-615 (2007).

5. Chen, G., Paronyan, T. M., Pigos, E. M. \& Harutyunyan, A. R. Enhanced gas sensing in pristine carbon nanotubes under ultraviolet light illumination. Sci. Rep. 2 (2012).

6. Xu, Z. \& Gao, C. Graphene chiral liquid crystals and macroscopic assembled fibres. Nat. Commun. 2, 571 (2011).

7. Lee, D. H. et al. Versatile Carbon Hybrid Films Composed of Vertical Carbon Nanotubes Grown on Mechanically Compliant Graphene Films. Adv. Mater. 22, 1247-1252 (2010).

8. Paul, R. K. et al. Synthesis of a Pillared Graphene Nanostructure: A Counterpart of Three-Dimensional Carbon Architectures. Small 6, 2309-2313 (2010).

9. Xiang, Q., Yu, J. \& Jaroniec, M. Synergetic Effect of MoS2 and Graphene as Cocatalysts for Enhanced Photocatalytic H2 Production Activity of TiO2 Nanoparticles. J. Am. Chem. Soc. 134, 6575-6578 (2012).

10. Guo, S. \& Sun, S. FePt Nanoparticles Assembled on Graphene as Enhanced Catalyst for Oxygen Reduction Reaction. J. Am. Chem. Soc. 134, 2492-2495 (2012).

11. Hata, K. et al. Water-assisted highly efficient synthesis of impurity-free singlewalled carbon nanotubes. Science 306, 1362-1364 (2004).

12. Robertson, J. Growth of nanotubes for electronics. Materials today 10, 36-43 (2007).

13. Futaba, D. N. et al. Shape-engineerable and highly densely packed single-walled carbon nanotubes and their application as super-capacitor electrodes. Nature Mater. 5, 987-994 (2006).

14. Li, X. et al. Large-Area Synthesis of High-Quality and Uniform Graphene Films on Copper Foils. Science 324, 1312-1314 (2009).

15. Paronyan, T. M., Pigos, E. M., Chen, G. \& Harutyunyan, A. R. Formation of Ripples in Graphene as a Result of Interfacial Instabilities. ACS Nano 5, 9619-9627 (2011).

16. Lee, Y. et al. Wafer-Scale Synthesis and Transfer of Graphene Films. Nano Lett. 10, 490-493 (2010).

17. Berciaud, S., Ryu, S., Brus, L. E. \& Heinz, T. F. Probing the Intrinsic Properties of Exfoliated Graphene: Raman Spectroscopy of Free-Standing Monolayers. Nano Lett. 9, 346-352 (2009).

18. Jorio, A., Dresselhaus, M. S. \& Saito, R. Raman Spectroscopy in Graphene Related Systems: Carbon Nanotubes, Nanographite and Graphene. (Wiley-VCH, 2011).

19. Ferrari, A. C. et al. Raman Spectrum of Graphene and Graphene Layers. Phys. Rev. Lett. 97, 187401 (2006).

20. Li, X., Cai, W., Colombo, L. \& Ruoff, R. S. Evolution of Graphene Growth on Ni and $\mathrm{Cu}$ by Carbon Isotope Labeling. Nano Lett. 9, 4268-4272 (2009).

21. Suk, J. W. et al. Transfer of CVD-Grown Monolayer Graphene onto Arbitrary Substrates. ACS Nano 5, 6916-6924 (2011).

22. Talapatra, S. et al. Direct growth of aligned carbon nanotubes on bulk metals. Nature Nanotech. 1, 112-116 (2006).

23. Hiraoka, T. et al. Synthesis of Single- and Double-Walled Carbon Nanotube Forests on Conducting Metal Foils. J. Am. Chem. Soc. 128, 13338-13339 (2006).

24. Reina, A. et al. Large Area, Few-Layer Graphene Films on Arbitrary Substrates by Chemical Vapor Deposition. Nano Lett. 9, 30 (2009).

25. Horcas, I. et al. WSXM: A software for scanning probe microscopy and a tool for nanotechnology. Rev. Sci. Inst. 78, 013705-013705 (2007).

26. Lee, C., Wei, X., Kysar, J. W. \& Hone, J. Measurement of the Elastic Properties and Intrinsic Strength of Monolayer Graphene. Science 321, 385-388 (2008).

27. Yoo, E. et al. Enhanced electrocatalytic activity of Pt subnanoclusters on graphene nanosheet surface. Nano Lett. 9, 2255-2259 (2009).

28. Luo, Z. et al. Size-Selective Nanoparticle Growth on Few-Layer Graphene Films. Nano Lett. 10, 777-781 (2010).

29. Lucchese, M. M. et al. Quantifying ion-induced defects and Raman relaxation length in graphene. Carbon 48, 1592-1597 (2010).

30. Bayer, B. C. et al. In-situ study of growth of carbon nanotube forests on conductive CoSi[sub 2] support. J. Appl. Phys. 109, 114314-114317 (2011).

31. Song, J. Y., Lee, H. H., Wang, Y. Y. \& Wan, C. C. Two- and three-electrode impedance spectroscopy of lithium-ion batteries. Journal of Power Sources 111, 255-267 (2002)

32. Vadivel Murugan, A., Reddy, M. V., Campet, G. \& Vijayamohanan, K. Cyclic voltammetry, electrochemical impedance and ex situ X-ray diffraction studies of 
electrochemical insertion and deinsertion of lithium ion into nanostructured organic-inorganic poly(3,4-ethylenedioxythiophene) based hybrids. Journal of Electroanalytical Chemistry 603, 287-296 (2007).

33. Andrews, R., Jacques, D., Rao, A. \& Derbyshire, F. Continuous production of aligned carbon nanotubes: a step closer to commercial realization. Chem. Phys. Lett. 303 (1999).

\section{Acknowledgements}

We thank T.M. Paronyan for preparing some of the graphene samples, Q. Xu for AFM measurements, Y.Li for fabrication of the trenched quartz substrates, and J. Katoch for preparation of the exfoliated graphene on $\mathrm{SiO}_{2}$ substrates. M.I. was supported by the National Science Foundation grant 0955625, and T.F.H. was supported by the Department of Energy, Office of Basic Energy Sciences grant DE-FG02-07ER15842. This research was supported by the Honda Research Institute, USA Inc.

\section{Author contributions}

R.R. designed and carried out the synthesis and characterization experiments, G.C. performed the resistivity measurements, A.L.M.R. and K.K. performed the EIS measurements, M.I. and T.F.H. assisted with supported and suspended graphene, P.M.A. guided the EIS measurements and helped with data analysis, A.R.H. initiated and guided the overall research. R.R., A.L.M.R., T.F.H., P.M.A. and A.R.H. wrote the paper.

\section{Additional information}

Supplementary information accompanies this paper at http://www.nature.com/ scientificreports

Competing financial interests: The authors declare no competing financial interests.

License: This work is licensed under a Creative Commons

Attribution-NonCommercial-NoDerivs 3.0 Unported License. To view a copy of this license, visit http://creativecommons.org/licenses/by-nc-nd/3.0/

How to cite this article: Rao, R. et al. Graphene as an atomically thin interface for growth of vertically aligned carbon nanotubes. Sci. Rep. 3, 1891; DOI:10.1038/srep01891 (2013). 